\title{
Dermatite factícia desencadeada pela síndrome de Münchausen
}

\author{
Factitial dermatitis as a consequence of Munchausen syndrome
}

\author{
Náiade Maria Rêgo e Silva ${ }^{1}$ \\ Esther Bastos Palitot ${ }^{3}$ \\ Samir de Figueiredo Azzouz ${ }^{5}$
}

\author{
Giannina Wanderley Petrucci ${ }^{2}$ \\ Mohamed Arbaqui Azzouz ${ }^{4}$
}

\begin{abstract}
Resumo: Dermatite factícia refere-se à condição em que as lesões cutâneas são autoinduzidas e resultam de algum distúrbio psiquiátrico. As lesões podem se apresentar de diversas formas e ser produzidas por grande variedade de meios. Apresenta-se um caso raro de paciente com dermatite factícia desencadeada pela síndrome de Münchausen, que se refere a indivíduos que intencionalmente produzem sintomas físicos com o intuito de atender a uma necessidade de receber atendimento médico frequente.
\end{abstract}

Palavras-chave: Dermatite; Psicopatologia; Síndrome de Münchausen

\begin{abstract}
Factitial dermatitis is a condition in which skin lesions are self-inflicted as the result of a psychiatric disorder. The lesions may be presented in various forms and produced by a wide variety of means. This report refers to the case of a patient with factitial dermatitis triggered by Munchausen syndrome, which refers to individuals who intentionally provoke physical symptoms in themselves in order to satisfy a need to receive frequent medical attention.

Keywords: Dermatitis; Psychopathology; Munchausen syndrome
\end{abstract}

\section{INTRODUÇÃO}

A dermatite factícia refere-se à condição em que lesões cutâneas são autoinduzidas, sendo resultado ou manifestação de algum distúrbio psiquiátrico. As lesões podem se apresentar de diversas formas ou padrões e, geralmente, situam-se em locais de fácil alcance às mãos do paciente. São produzidas por uma grande variedade de meios, como unhas, objetos pontiagudos, substâncias químicas, entre outros. ${ }^{1}$ Considera-se o diagnóstico de dermatite factícia quando as hipóteses de doenças orgânicas são excluídas. Em raros casos, essa dermatite pode estar associada à síndrome de Münchausen, que se refere a indivíduos que intencionalmente produzem ou apresentam sin- tomas físicos com o intuito de receber atendimento hospitalar frequente. ${ }^{2}$ Descrita pela primeira vez em 1951, a síndrome de Münchausen tem como critérios diagnósticos definidores a produção ou simulação intencional de sinais e sintomas predominantemente físicos, a necessidade do paciente de sentir-se doente e a ausência de incentivos externos para seu comportamento (ganho econômico, fuga de responsabilidade legal ou melhora de bem-estar físico). Pelas próprias características do quadro, poucos pacientes são atendidos por um psiquiatra. A maioria deles perambula por inúmeros hospitais e é atendida nos diferentes serviços de clínica e de cirurgia de emergência, sendo

\footnotetext{
Recebido em 14.08.2008.

Aprovado pelo Conselho Editorial e aceito para publicação em 30.10.2008

Trabalho realizado na Universidade Federal da Paraíba (UFPB) - João Pessoa (PB), Brasil.

Conflito de interesse: Nenhum / Conflict of interest: None

Suporte financeiro: Nenhum / Financial funding: None

Acadêmica de Medicina - Universidade Federal da Paraíba (UFPB) - João Pessoa (PB), Brasil.

Acadêmica de Medicina - Universidade Federal da Paraíba (UFPB) - João Pessoa (PB), Brasil.

Mestre com área de concentração em Dermatologia; professora de Dermatologia da Universidade Federal da Paraíba (UFPB) - João Pessoa (PB), Brasil.

Mestre com área de concentração em Dermatologia; professor de Dermatologia da Universidade Federal da Paraíba (UFPB) - João Pessoa (PB), Brasil.

Acadêmico de Medicina - Faculdade de Medicina Souza Marques (FMSM) - Rio de Janeiro (RJ), Brasil. 


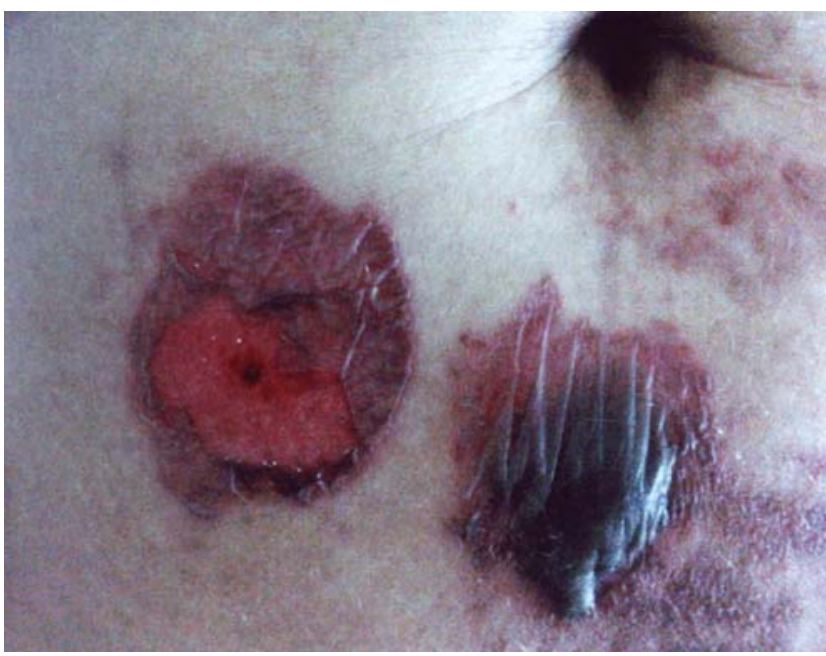

Figura 1: Lesões bolhosas e exulceradas em região periumbilical

o diagnóstico dificultado pelo desconhecimento dos profissionais de saúde a respeito da síndrome. 3,4 Neste relato, descreve-se e discute-se um caso raro de uma paciente com dermatite factícia desencadeada pela síndrome de Münchausen.

\section{RELATO DO CASO}

Paciente do sexo feminino, 36 anos, solteira, doméstica, procurou serviço médico em um hospital de emergência, referindo lesões dermatológicas há cerca de seis meses e atribuindo a seu patrão a autoria das mesmas. Ao exame, apresentava lesões bolhosas exulceradas, bem regulares, distribuídas pelos braços, coxas e tronco (Figura 1). Afirmou já ter sido atendida em outros hospitais, um dos quais deu o diagnóstico clínico de pênfigo vulgar. Posteriormente, a paciente foi submetida à biópsia da lesão, cujo laudo do histopatológico foi compatível com uma dermatite de contato irritativa por um agente causal inespecífico (Figura 2). Como a paciente se apresentava muito inquieta e confusa, foi submetida a uma avaliação psiquiátrica, em que se constatou que as lesões eram decorrentes da aplicação de uma substância cáustica à pele e eram provocadas pela própria paciente. Concluiu-se que se tratava de uma dermatite factícia desencadeada pela síndrome de Münchausen. A paciente recebeu tratamento tópico sintomático das lesões e foi encaminhada para acompanhamento psiquiátrico.

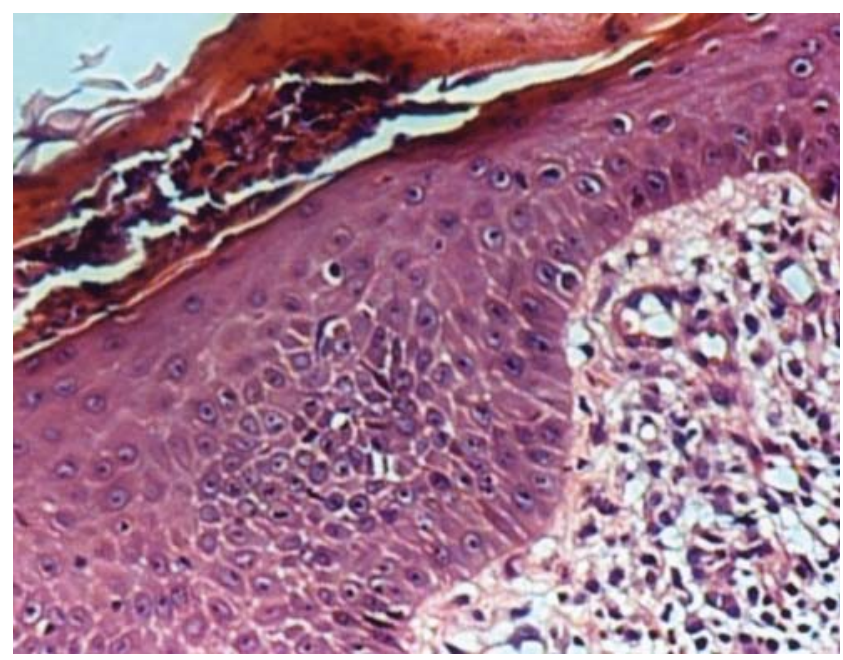

Figura 2: Epiderme paraceratótica com exsudação neutrofílica intracórnea. $\mathrm{Na}$ derme, infiltrado inflamatório misto, monomorfonuclear e polimorfonuclear, principalmente perivascular (HE 100x)

\section{DISCUSSÃO}

A paciente descrita no caso mimetizava uma enfermidade dermatológica por meio da aplicação de hidróxido de sódio (soda cáustica) sobre a pele. Negava peremptoriamente a sua participação nas lesões, chegando a acusar o seu patrão de agressão física e responsabilizando-o, dessa forma, pela autoria das lesões. A paciente teve passagens por vários hospitais, com o mesmo quadro clínico, tendo sido aventadas outras hipóteses diagnósticas. Diante da recorrência do quadro e do estado de inquietação em que a paciente se encontrava, no último atendimento, solicitou-se avaliação psiquiátrica; após várias consultas, foi dado o diagnóstico de dermatite factícia desencadeada pela síndrome de Münchausen. O comportamento da paciente é bem característico, sendo motivado por uma necessidade intrínseca de receber atenção, principalmente, do ponto de vista médico-hospitalar.

A associação das duas condições citadas, além de ser rara, muitas vezes, não é diagnosticada, devido ao desconhecimento dos profissionais de saúde a respeito do tema. O manejo clínico desses pacientes é dificultado pela baixa adesão ao tratamento e eles devem ser observados de forma cautelosa, para que novas lesões não sejam provocadas durante o acompanhamento médico. ${ }^{5,6}$ É imprescindível a abordagem do ponto de vista psiquiátrico, que é a principal responsável pela cura completa dos pacientes, evitandose, assim, frequentes recidivas. ${ }^{6,7}$ 


\section{REFERÊNCIAS}

1. Sun DK, Siegel DM. A nonhealing ulcer on the face. Arch Fam Med. 2000;9:787-9.

2. Zalewska A, Kondras K, Narbutt J, Sysa-Jedrzejowska A. Dermatitis artefacta in a patient with paranoid syndrome. Acta Derm Venereol. 2007; 16:37-9.

3. Menezes APT, Holanda EM, Silveira VAL, Oliveira KCS, Oliveira FGM. Síndrome de Münchausen: relato de caso e revisão da liter atura. Rev Bras Psiquiatr. 2002;24:83-5.

4. Gattaz WF, Dressing H, Hewer W, Nunes P. Síndrome de Münchausen: diagnóstico e manejo clínico. Rev Assoc Med Bras. 2003;49:220-4.

5. Rosen T, Chen M. Factitious disease of the breast of a male due to injection of liquid plastic. Int J Dermatol. 2001;40:743-6.
6. Aleem A, Ajarim DS. Münchausen syndrome presenting as immunodeficiency: a case report and review of literature. Ann Saudi Med. 1995;15:404-6.

7. Obasi OE, Naguib M. Dermatitis artefacta: a review of 14 cases. Ann Saudi Med. 1999;19:223-7.

ENDEREÇO PARA CORRESPONDÊNCIA / MAILING ADDRESS:

Mohamed Azzouz / Esther Palitot

Rua Augusto dos Anjos, 35 - Centro.

58030090 João Pessoa, PB

Tel.:/Fax: 833241 8303, 91570502 e 91067840

E-mail:azzouz@globo.com /

esther_palitot@hotmail.com

Como citar este artigo/How to cite this article: Silva NMR, Petrucci GW, Palitot EB, Azzouz MA, Azzouz SF. Dermatite factícia desencadeada pela síndrome de Münchausen. An Bras Dermatol. 2010;85(1):77-80. 